\title{
Inversão gravimétrica 2D empregando o método estocástico PSO
}

\author{
Leonardo M. Batista $^{1}$, Daniel E. Revelo ${ }^{1}$, Milton J. Porsani ${ }^{1}$ \\ ${ }^{1} \mathrm{CPGG} / \mathrm{UFB}$ \\ Copyright 2018, SBGf - Sociedade Brasileira de Geofísica. \\ Este texto foi preparado para a apresentação no VIII Simpósio Brasileiro de Geofísica, \\ Salinópolis, 18 a 20 de setembro de 2018 . Seu conteúdo foi revisado pelo Comitê \\ Técnico do VIII SimBGf, mas não necessariamente representa a opinião da SBGf ou de \\ seus associados. É proibida a reprodução total ou parcial deste material para propósitos \\ comerciais sem pr'evia autorização da SBGf.
}

\section{Resumo}

A inversão de dados gravimétricos para a estimativa das profundidades da interface entre uma dada bacia sedimentar e o embasamento representa uma aplicação importante das técnicas de inversão não linear. Uma abordagem comum para aproximar este problema consiste na discretização da bacia utilizando polígonos (ou outras geometrias), e então, recursivamente solucionar o problema inverso não linear através de métodos de escopo local. No entanto, estes algoritmos têm uma alta dependência da informação a priori, que deve ser empregada a fim de evitar os mínimos locais e obter uma solução aceitável. Um outro grupo de algoritmos de otimização podem ser aplicados na inversão gravimétrica, os quais correspondem aos métodos de escopo global. Levando em conta as vantagens descritas na literatura sobre esses últimos métodos, tem sido escolhido o método de otimização por enxame de partículas (PSO - Particle Swarm Optimization) para ser aplicado no problema gravimétrico. A fim de avaliar o desempenho do PSO no esquema de otimização, um teste inicial empregando a função analítica Schwefel foi realizado. Em seguida, o PSO foi empregado na inversão de dados gravimétricos sintéticos 2D, os resultados obtidos deixam em evidência a possibilidade de realizar uma inversão eficiente e com baixa incerteza no modelo estimado. Adicionalmente, é demonstrada a robustez do PSO através de um teste de inversão da anomalia gravimétrica contaminada com ruído branco (Gaussiano).

\section{Introdução}

Os problemas de otimização geofísica geralmente são não lineares, multidimensionais e caracterizados por funções objetivas complexas com muitos mínimos locais. Existem duas estratégias para enfrentar esses problemas: os métodos determinísticos, baseados no computo do gradiente da função objetivo, e os algoritmos Monte Carlo (MC) ou estocásticos, que realizam uma busca direta no espaço de soluções. Esses últimos, são atrativos devido à não demanda do cálculo de derivadas, assim como ao bom desempenho na exploração do espaço de modelos. No entanto, os esquemas de inversão estocásticos apresentam uma alta demanda computacional quando a etapa da modelagem direta é custosa, além disso sofrem do problema da maldição de dimensionalidade, relacionada à falta de convergência do método no caso de problemas com grande quantidade de incógnitas.

A primeira aplicação dos métodos $M C$ em geofísica foi apresentada por Press (1968) na área de sismologia, onde foram invertidos tempos de trânsito de ondas de corpo. Desde então, os métodos MC foram aplicados para resolver um número crescente de problemas geofísicos. Uma propriedade atraente dos métodos MC é que eles não precisam da suposição de linearidade do problema inverso, sendo um aspecto conveniente para os problemas geofísicos, que na maioria dos casos são não lineares ( $p$. ex., a estimativa de velocidades sísmicas a partir de formas de onda sísmica). A necessidade de métodos de otimização mais potentes e o crescente poder computacional fornecido pelos computadores modernos incentivaram a criação e o desenvolvimento de vários métodos heurísticos parcialmente estocásticos, muitos dos quais foram testados com sucesso no campo geofísico. Alguns desses métodos são: o algoritmo de recozimento simulado (SA - Simulated Annealing), o algoritmo genético (GA - Genetic Algorithm) (Holland, 1975), a otimização por enxames de partículas (PSO - Particle Swarm Optimization) (Kennedy e Eberhart, 1995), entre outros.

É interessante analisar o desempenho dos diferentes métodos $M C$ em diferentes cenários, sendo uma comparação interessante a apresentada por Ingber e Rosen (1992). Em geral, os resultados obtidos na comparação dos métodos MC, não podem ser estendidos para tirar conclusões gerais. Isso ocorre porque o resultado de uma única comparação depende da complexidade do problema a ser resolvido, bem como a implementação particular do algoritmo e a escolha dos valores dos parâmetros de controle. Assim, no presente trabalho aplicamos o PSO num problema geofísico clássico: a inversão gravimétrica, abordando os casos livre de ruído e contaminado com ruído branco. A inversão gravimétrica é uma ferramenta da geofísica aplicada para analisar a estrutura das bacias sedimentares na exploração mineral, hidrogeologia, etc (Telford et al., 1976).

O problema de inverter as anomalias gravimétricas é linear quando uma dada geometria para os corpos em estudo é fornecida e as densidades correspondentes são os parâmetros a serem estimados. Por outro lado, o problema inverso não é linear quando a geometria dos corpos é tratada como desconhecida, fazendo algumas suposições sobre os valores das densidades correspondentes. Entre as técnicas não lineares, a determinação do relevo duma bacia sedimentar é uma tarefa relativamente comum (Chakravarthi et al., 2001). A maneira clássica de lidar com esse problema é através da otimização não linear, onde as incógnitas são a profundidade da bacia ou a profundidade e alguns parâmetros adicionais são levado em consideração, como a variação da densidade dos sedimentos. Neste trabalho, o método PSO é aplicado na inversão gravimétrica, tendo como incógnitas as profundidades dos sedimentos que compõem a bacias sedimentares. 


\section{Inversão e Modelagem Gravimétrica 2D}

A inversão gravimétrica do relevo do embasamento em bacias sedimentares, consiste na inversão de um perfil da bacia em estudo. Esse esquema de inversão, baseado na minimização de uma função que compara as anomalias gravimétricas observadas e calculadas, necessita do conhecimento de um operador de modelagem. Na Figura 1, apresenta-se o esquema do acréscimo de prismas retangulares (Chakravarthi, 1995; Barbosa et al., 1997), o qual é empregado na modelagem gravimétrica 2D para o caso de densidade constante. Assim, no presente estudo será utilizado esse esquema, além de restringir o problema da inversão ao caso determinado, no qual o número de parâmetros é igual ao número de prismas utilizados para definir a bacia sedimentar.

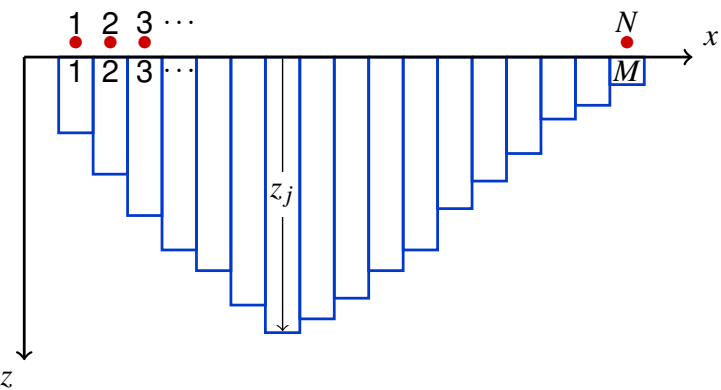

Figura 1: Modelagem bidimensional de uma bacia sedimentar por prismas retangulares retos. $M$ representa 0 número de prismas e $N$ os pontos de observações.

Uma maneira comum de resolver o problema inverso gravimétrico, e pelo tanto, estimar o modelo de profundidades $\mathbf{z}$, é aquela que baseia-se na minimização da função de objetivo:

$$
c^{g}(\mathbf{z})=\frac{1}{N}\left\|\Delta \mathbf{g}^{o b s}-\Delta \mathbf{g}(\mathbf{z})\right\|_{2}^{2},
$$

sendo $\Delta \mathbf{g}^{o b s}$ o vetor das anomalias gravimétricas observadas, e $\Delta \mathbf{g}(\mathbf{z})$ o vetor das anomalias calculadas para o modelo $\mathbf{z}$.

De acordo com Barbosa et al. (1997), o valor $\Delta \mathbf{g}(\mathbf{z})$ é calculado considerado o conjunto de $N$ anomalias gravimétricas medidas ao longo de um perfil nos pontos de observações $p_{i}\left(x_{i}, z_{i}\right)$, isso adotando-se uma partição da bacia em $M$ prismas retangulares com contraste de densidade constante conhecida $\Delta \rho=\rho_{s}-\rho_{b}$ (os índices $s$ e $b$ se referem aos sedimentos e basamentos, respectivamente). Assim, $\Delta g$ em cada ponto de observação será dada por:

$$
\Delta g_{i}=\sum_{j=1}^{M} F\left(\Delta \rho_{j}, z_{j}, r_{i j}\right), \quad i=1,2, \ldots, N,
$$

sendo $F\left(\Delta \rho_{j}, z_{j}, r_{i j}\right)$ a função que resolve o problema direto para um prisma individual no ponto de observação $i, \Delta \rho_{j}$ é o contraste de densidade do j-ésimo prisma, $z_{j}$ é a profundidade do $j$-ésimo prisma, e $r_{i j}$ é o vetor posição horizontal entre o $i$-ésimo ponto e o $j$-ésimo prisma.

Considerando um prisma retangular 2D (Figura 2) com valor de densidade constante $(\rho)$, a atração gravitacional $F$ gerada por este prisma no ponto $P(x, z)$ pode ser calculada pela expressão (Barbosa e Silva, 1994; Telford et al., 1976):

$$
\begin{aligned}
F & =F\left(x, z, x_{m}, x_{M}, z_{t}, z_{b}, \rho\right) \\
& =G \rho\left[A \ln \frac{A^{2}+D^{2}}{A^{2}+C^{2}}-B \ln \frac{B^{2}+D^{2}}{B^{2}+C^{2}}\right. \\
- & \left.2 C\left(\arctan \frac{A}{C}-\arctan \frac{B}{C}\right)+2 D\left(\arctan \frac{A}{D}-\arctan \frac{B}{D}\right)\right],
\end{aligned}
$$

onde, $A=x-x_{m}, B=x-x_{M}, C=z-z_{t}, D=z-z_{b}$ e $G$ a constante gravitacional de Newton.

O problema inverso não linear estabelecido na Eq. 1 é mal posto, uma vez que existem infinitas soluções (z) que se encaixam nos dados observados dentro dos mesmos limites de erro. Assim, diversas técnicas iterativas de otimização local, tais como Levenberg-Marquard (Marquardt, 1963), Gauss-Newton e métodos QuaseNewton (Nocedal e Wright, 1999) tem sido utilizadas na solução desse problema, além de utilizar processos de regularização. No entanto, o sucesso desses algoritmos depende do modelo inicial, assim como da informação a priori adotada. Um outro tipo de estratégia de otimização global podem ser empregadas na solução do problema gravimétrico, trata-se dos algoritmos estocásticos. Esse tipo de métodos realizam pesquisas inteligentes sobre o espaço dos modelos a fim de determinar a direção das regiões dos valores mínimos da função de custo a ser minimizada.

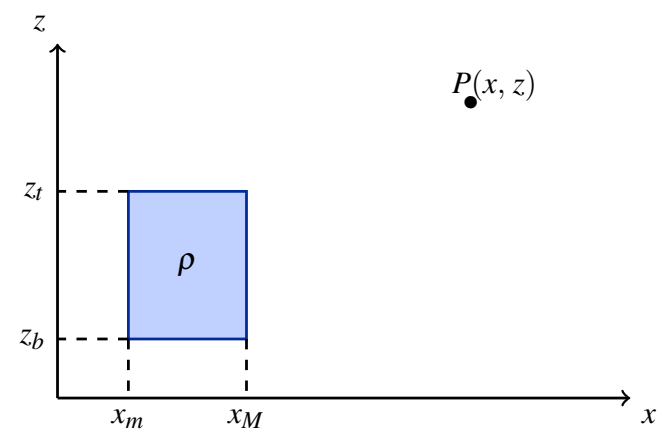

Figura 2: Situação esquemática bidimensional dum prisma retangular e, o ponto $P(x, z)$ onde será avaliada a anomalia gravimétrica. $\rho$ : densidade, $x_{m}$ : mínima coordenada em $x$, $x_{M}$ : máxima coordenada em $x, z_{t}$ : coordenada em $z$ do lado superior do prisma, $z_{b}$ : coordenada em $z$ do lado inferior do prisma.

\section{Otimização por enxame de partículas}

Proposto por Kennedy e Eberhart (1995), o método de otimização por enxame de partículas (PSO - Particle Swarm Optimization) é uma técnica de otimização global inspirada no comportamento social de organismos biológicos, pelo qual pode ser incluído no conjunto de algoritmos evolutivos. Neste algoritmo de busca, um enxame de partículas (ou modelos) explora o espaço de possíveis soluções para otimizar uma determinada função de custo, que em nosso caso está relacionada ao problema de inversão gravimétrica (Eq. 1). No início do PSO, as partículas do enxame são inicializadas em posições aleatórias no espaço de busca segundo uma distribuição de probabilidade uniforme. 
Em seguida, a cada iteração $k$, as velocidades $\left(v_{i}\right)$ e as posições $\left(x_{i}\right)$ das partículas são atualizadas de acordo com as seguintes fórmulas (Figura 3):

$$
\begin{aligned}
v_{i}(k+1) & =\omega \times v_{i}(k)+a_{1} \times a_{l o c} \times \frac{\left(g_{i}-x_{i}(k)\right)}{\Delta t} \\
& +a_{2} \times a_{\text {glob }} \times \frac{\left(G-x_{i}(k)\right)}{\Delta t} \\
x_{i}(k+1) & =x_{i}(k)+v_{i}(k+1) \Delta t,
\end{aligned}
$$

onde, $\omega$ a inércia, $a_{l o c}$ e $a_{g l o}$ as acelerações local e global, respectivamente, $a_{1}$ e $a_{2}$ os números randômicos entre $0 \mathrm{e}$ 1 , e $\Delta t$ é o espaço de tempo.

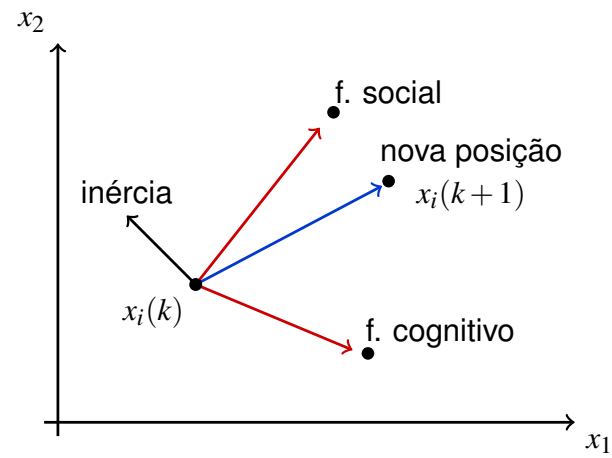

Figura 3: Exemplo de movimento de uma partícula em um espaço de busca com 2 dimensões.

Para evitar o aumento abrupto da velocidade do PSO original proposto por Kennedy, passou-se a limitá-la por um valor máximo (Engelbrecht, 2007), usando a seguinte expressão:

$$
v_{i j}(l)= \begin{cases}v_{i j}(l), & \text { se }\left|v_{i j}(l)\right|<v_{\max , j} \\ \operatorname{signal}\left(v_{i j}(l)\right) \times v_{\max , j}, & \text { se }\left|v_{i j}(l)\right| \geq v_{\max , j},\end{cases}
$$

sendo, $l=k+1$, e $v_{i j}(k+1)$ a velocidade de cada partícula para cada dimensão usando a Eq. 4. A estratégia geralmente adotada para escolher cada componente $v_{\max , j}$ do vetor velocidade máxima é defini-lo como uma fração do espaço de busca (Engelbrecht, 2007), da forma

$$
v_{\max , j}=\alpha \times\left(x_{\max , j}-x_{\min , j}\right),
$$

em que $x_{\max , j}$ e $x_{\min , j}$ são os valores máximos e mínimos do espaço de busca, $\alpha$ é um parâmetro tal que $\alpha \in(0,1]$.

A fim de impor que as partículas façam a busca dentro do espaço de soluções de interesse durante o procedimento de otimização, várias condições de contorno são atualmente usadas no algoritmo PSO. No presente trabalho, devido ao bom desempenho apresentado, o limite refletivo tem sido selecionado, dita condição de fronteira volta à partícula ao espaço de busca nessa dimensão e o sinal do componente da velocidade é invertido.

Para garantir a convergência, Clerc (1999) desenvolveu uma abordagem para equilibrar o compromisso entre exploração e explotação do PSO, na qual a equação da velocidade é modificada para:

$$
\begin{aligned}
v_{i}(k+1) & =\phi \times\left(v_{i}(k)+a_{1} \times a_{l o c} \times\left(g_{i}-x_{i}(k)\right)\right. \\
& \left.+a_{2} \times a_{g l o b} \times\left(G-x_{i}(k)\right)\right) \\
x_{i}(k+1) & =x_{i}(k)+v_{i}(k+1),
\end{aligned}
$$

onde, $\phi$ é um termo de constrição que depende das duas acelerações:

$$
\phi\left(a_{l o c}, a_{g l o b}\right)=\frac{2}{\left|2-a_{l o c}-a_{g l o b}-\sqrt{\left(a_{l o c}+a_{g l o b}\right)^{2}-4\left(a_{l o c}+a_{g l o b}\right)}\right|} .
$$

Essa última versão do PSO será empregada na solução do problema inverso aplicado sobre dados gravimétricos. No entanto, é importante mencionar que devido ao problema da granularidade presente no modelo estimado pelo PSO, uma condição de suavização para o relevo do embasamento deve ser empregada, a qual pode ser realizada através de um filtro de média móvel:

$$
z_{j}=\frac{\sum_{k=-n}^{n} z_{j+k}}{2 n+1},
$$

onde, $z_{j}$ a amplitude a ser determinada e $n$ a amplitude da janela aplicada.

\section{Experimentos numéricos}

Nesta seção são apresentados os resultados obtidos na aplicação do método PSO para a determinação do mínimo global da função Schwefel e posteriormente aplicado na inversão de dados gravimétricos 2D, tanto livres de ruído, assim como com ruído branco.

\section{Teste com a função Schwefel}

Tendo como principal finalidade analisar o comportamento do PSO quando o número de parâmetros desconhecidos aumenta, foi utilizada a função analítica Schwefel, que apresenta o mínimo global na borda do espaço de busca e vários mínimos distribuídos irregularmente (Figura 4). Como diretriz, seguimos estritamente as considerações (i.e., número de dimensões, critério de convergência, etc) do trabalho de Sajeva et al. (2017). O critério de convergência foi selecionado para quando o PSO estima um modelo $\mathbf{x} \in[-5,5]^{n}$ tal que:

$$
\varepsilon \leq \sqrt{\frac{\sum_{i=1}^{n}\left(x_{i}-x_{i}^{g l o b}\right)^{2}}{n}},
$$

sendo $\mathbf{x}^{g l o b}$ o mínimo global, e a precisão $\varepsilon$ é estabelecida como 0.05. A fim de obter resultados estatisticamente significativos, realizamos 100 testes com o algoritmo PSO, para cada dimensão do espaço dos modelos. Os parâmetros de controle do algoritmo PSO foram $a_{l o c}=1.2 \mathrm{e}$ $a_{\text {glob }}=2.9$, garantindo que $a_{l o c}>a_{\text {glob }}$ para assim aumentar a exploração do espaço de modelos, $v_{\max }=5 \mathrm{e}$, o tamanho da população aumentando linearmente da forma $100 n$.

$\mathrm{Na}$ Figura $5 \mathrm{a}$, é apresentada a porcentagem de sucesso calculado no conjunto de 100 testes numa faixa de dimensões do espaço dos modelos que varia de 2 a 60 . A Figura $5 \mathrm{~b}$ mostra a número de modelos avaliados quando a dimensão $n$ aumenta para a função Schwefel. O algoritmo PSO exibe uma pobre escalabilidade com o aumento de $n$, sem uma tendência clara de convergência associada à crescente complexidade do problema. Em termos gerais o PSO com limite refletivo apresentou um bom desempenho para baixas dimensões, já para faixas superiores a $n=14$ a 
convergência é baixa, o qual pode ser resultado do número de partículas utilizadas e, ao fato de atingir um mínimo local prematuramente.

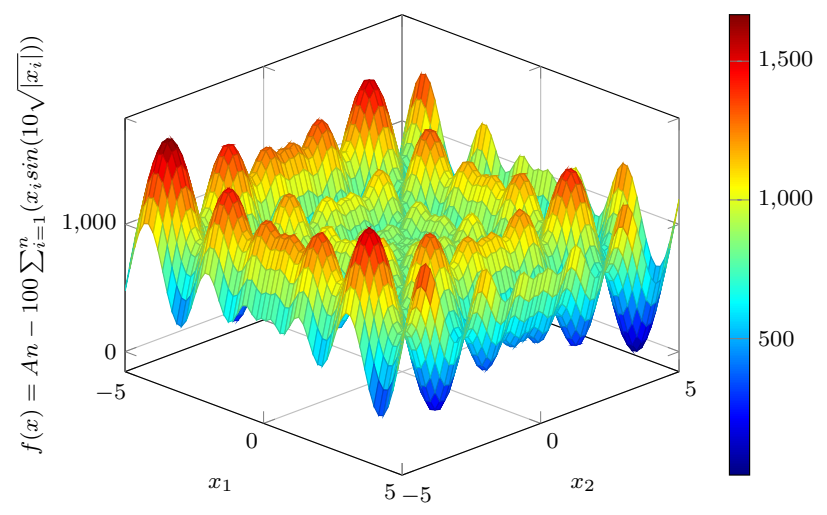

Figura 4: Função Schwefel.

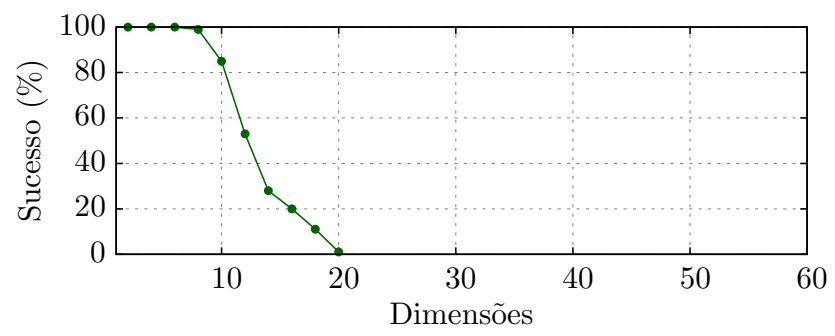

a) Número de experimentos bem sucedidos.

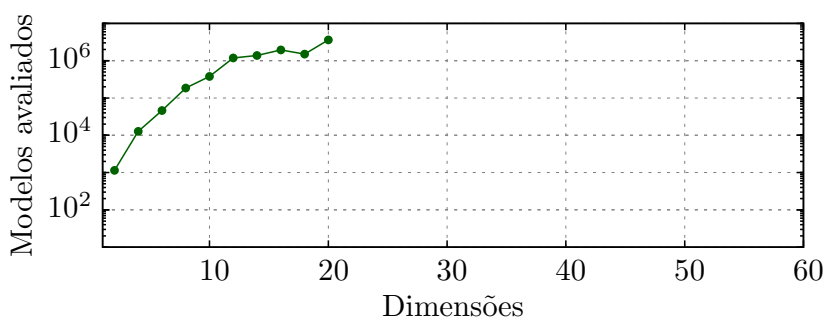

b) Número de modelos avaliados.

Figura 5: Resultados obtidos no teste aplicado sobre a função Schwefel.

\section{Inversão gravimétrica}

Para o problema de inversão gravimétrica 2D, os limites de busca para a profundidade de cada prisma são estimados da seguinte forma:

i Um modelo aproximado $\mathbf{z}^{0}$ é calculado usando a anomalia gravimétrica observada e aplicando a correção de Bouguer para placas, que no caso de um contraste de densidade constante (Barbosa et al., 1997) é dada por:

$$
z_{j}^{0}=\frac{\Delta g_{j}^{o b s}}{2 \pi G \Delta \rho},
$$

sendo $z_{j}^{0}$ a profundidade inicial do $j$-ésimo prisma (Figura 1), $\Delta g_{j}^{o b s}$ é a anomalia gravimétrica observada no centro do prisma, $G$ é a constante gravitacional universal, e $\Delta \rho$ é contraste de densidade constante, que no presente trabalho será um valor conhecido.

ii Com o objetivo de definir os limites no espaço de busca, fatores $k_{\min }<1.0$ e $k_{\max }>1.0$ são aplicados sobre o modelo aproximado $z_{j}^{0}$, obtendo o seguinte domínio para $\mathbf{z}$ :

$$
\mathbf{z} \in\left[k_{\min } \cdot \mathbf{z}^{0}, k_{\max } \cdot \mathbf{z}^{0}\right]
$$

sendo que $\mathbf{z}^{0}$ tem a mesma dimensionalidade do vetor dos parâmetros a serem estimados, $\mathbf{z}$.

A seguir são apresentados dois testes de aplicação do método PSO na determinação do relevo de embasamento: o primeiro caso trata-se dum problema inverso gravimétrico livre de ruído, já no segundo exemplo será realizada a inversão para um dado gravimétrico que tem sido contaminado com ruído Gaussiano. O modelo sintético da bacia é composto por 50 prismas retangulares com largura de $1.5 \mathrm{~km}$ e os seus topos coincidindo com o plano $z_{t}=0 \mathrm{~km}$ (Figura 6a). Utilizando a Eq. 2 e presumindo um valor constante no contraste de densidade $(\Delta \rho=$ $-0.25 \mathrm{~g} / \mathrm{cm}^{3}$ ), foi calculada a anomalia Bouguer em 50 estações regularmente espaçadas (Figura 6b). O espaço de busca foi gerado com $k_{\text {min }}=0.8$ e $k_{\max }=1.5$.

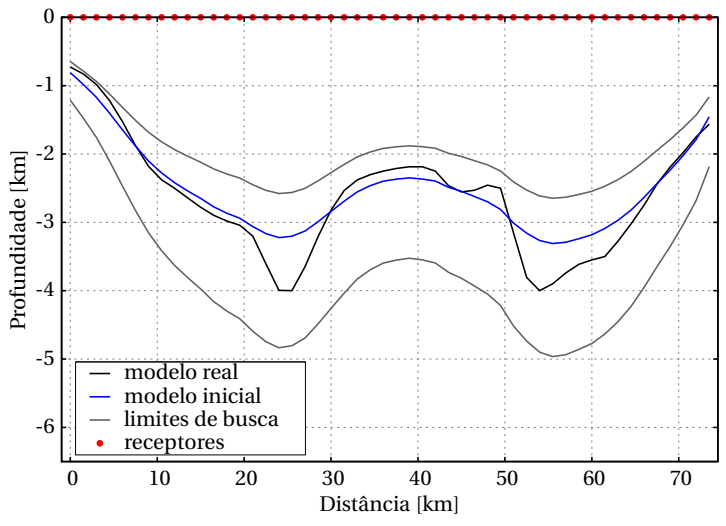

a) Modelo sintético da bacia sedimentar.

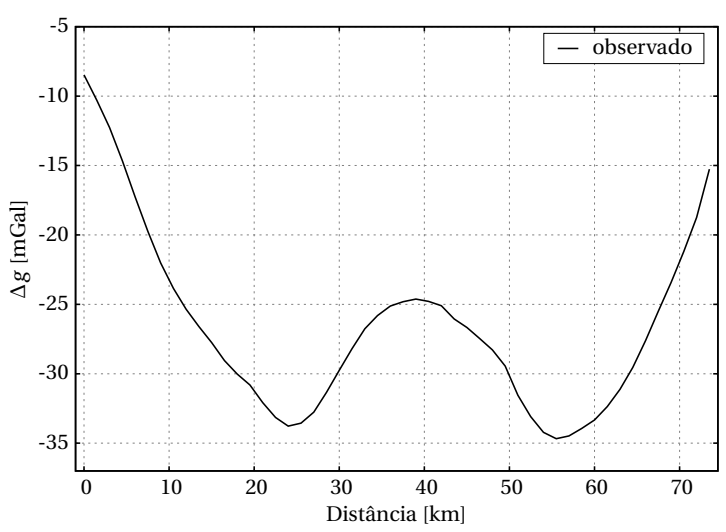

b) Anomalia observada.

Figura 6: Modelo verdadeiro e dados observados utilizados na inversão gravimétrica. 
Em relação com os parâmetros do algoritmo $\mathrm{PSO}$, foram mantidos os valores apresentados no teste com a função Schwefel, no entanto, o tamanho da população foi 250 e o número de iterações 300 . Como critério de parada foi utilizada a porcentagem de erro entre os parâmetros dos dados $\left(\varepsilon_{d}\right)$ :

$$
\varepsilon_{d}=\sqrt{\frac{\sum_{i=1}^{M}\left(g_{i}^{\text {obs }}-g_{i}^{\text {calc }}-\right)^{2}}{\sum_{i=1}^{M}\left(g_{i}^{\text {obs }}\right)^{2}}} \times 100 \% .
$$

Desta forma, se $\varepsilon_{d}$ é menor que o valor predefinido (2\%), o algoritmo PSO finaliza e o modelo nessa iteração será tomado como o melhor modelo estimado $\left(\mathbf{z}^{e s t}\right)$, do contrario, o processo é continuado até que o critério de parada seja alcançado. Determina-se também para efeito de comparação, a percentagem de erro entre os parâmetros dos modelos $\left(\varepsilon_{m}\right)$ :

$$
\varepsilon_{m}=\sqrt{\frac{\sum_{j=1}^{N}\left(z_{j}^{\text {verd }}-z_{j}^{\text {stt }}\right)^{2}}{\sum_{j=1}^{N}\left(z_{j}^{\text {verd }}\right)^{2}}} \times 100 \% .
$$

Nos dois casos a serem apresentados, dado gravimétrico com e sem ruído, será aplicado o filtro de média móvel com amplitude igual a 2, valor selecionado com base nos diversos experimentos realizados na presente pesquisa.

\section{Caso livre de ruído}

Na Figura 7a é apresentada a convergência do PSO com o passo das iterações, a curva mostra que o método atinge a região do $2 \%$ em menos de 140 iterações, o qual representa ao redor de 35250 modelagens. A Figura $7 \mathrm{~b}$ mostra o melhor modelo estimado pelo algoritmo PSO (com o filtro aplicado). No presente caso, o $\varepsilon_{m}$ obtido foi de $5.51 \%$.

\section{Caso com ruído Gaussiano}

No segundo exemplo sintético, um ruído branco aleatório de distribuição normal (5\%) foi adicionado às observações do exemplo anterior, a fim de analisar o desempenho do método PSO na inversão gravimétrica com anomalias sob o efeito do ruído. O restante dos parâmetros numéricos do experimento permaneceu inalterado.

Da Figura 8a pode-se observar que o PSO atinge um valor próximo de $2 \%$ para $\varepsilon_{d}$ em menos de 280 iterações, o qual é refletido em aproximadamente 70000 modelagens diretas. A partir do modelo estimado (Figura 8b) foi calculado $\varepsilon_{m}$, obtendo um valor de $5.67 \%$, o qual exibe a robustez do PSO na inversão de dados gravimétricos contaminados com ruído. É importante mencionar que o custo computacional aumento em relação ao caso da inversão com as anomalias livres de ruído, isto uma vez que o PSO requer um maior número de modelagens gravimétricas para atingir o critério de parada.

\section{Conclusões}

Neste trabalho foi apresentado o desempenho do método de otimização por enxame de partículas (PSO) para a localização do mínimo global de uma função analítica complexa (Schwefel), assim como para uma aplicação geofísica (inversão gravimétrica 2D). Para a função analítica Schwefel, observamos que a curva de convergência como função da dimensão do espaço do modelo tem uma tendência polinomial, mas com convergência até baixo número de dimensões. O método PSO foi implementado a fim de estimar o relevo duma bacia sedimentar 2D a partir de dados gravimétricos, onde foi utilizada uma abordagem clássica consistindo em descrever as bacias como agregados de prismas retangulares. $O$ desempenho do algoritmo PSO foi analisado em dois conjuntos de dados sintéticos (livre de ruído e com ruído Gaussiano), mostrando que o PSO fornece resultados consistentes com a geologia da bacia em estudo. A técnica PSO estudada mostrou-se como uma ferramenta poderosa e otimizada para estimar o relevo das bacias sedimentares 2D, razão pela qual podese pensar no uso do PSO para a estimativa de modelos iniciais no caso 3D. Em geral, a partir dos resultados obtidos nos experimentos realizados pode-se verificar que o PSO é uma boa alternativa na solução de problemas de otimização. Este algoritmo é de fácil implementação e possui custo computacional reduzido.

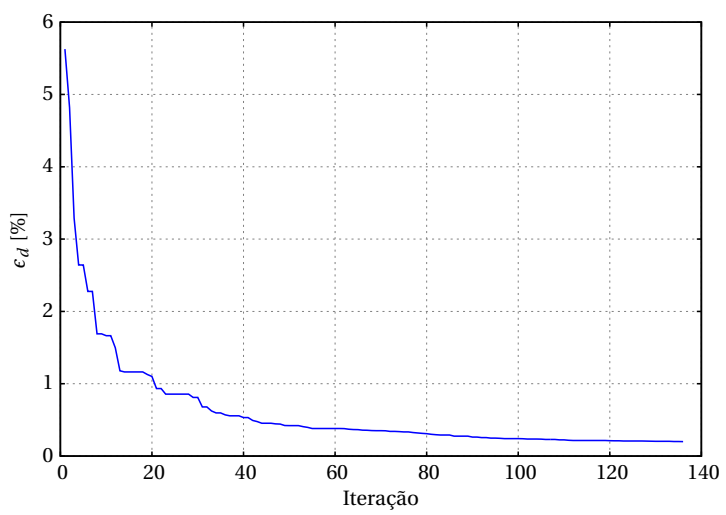

a) Convergencia do PSO.

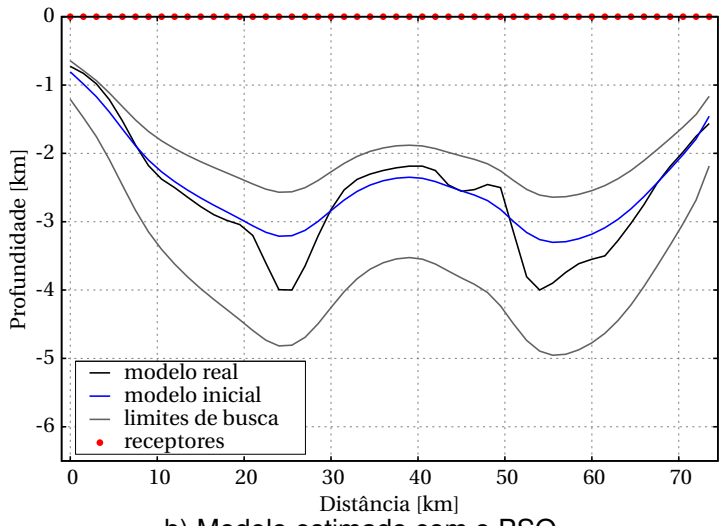

b) Modelo estimado com o PSO.

Figura 7: Resultados para o teste livre de ruido Gaussiano.

\section{Acknowledgements}

Os autores agradecem ao INCT-GP/CNPq e a CAPES pelo apoio financeiro no desenvolvimento do presente trabalho.

\section{Referências}

Barbosa, V. e J. Silva, 1994, Generalized compact gravity inversion.: Geophysics, 95, 57-68.

Barbosa, V., J. Silva, e W. Medeiros, 1997, Gravity inversion 


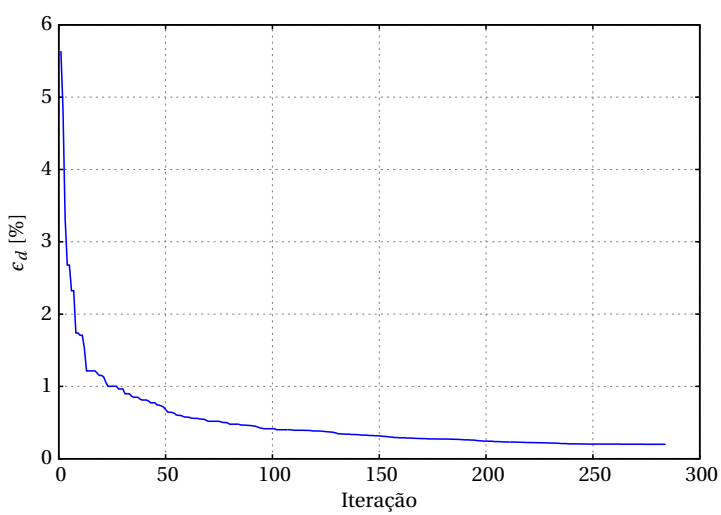

a) Convergencia do PSO.

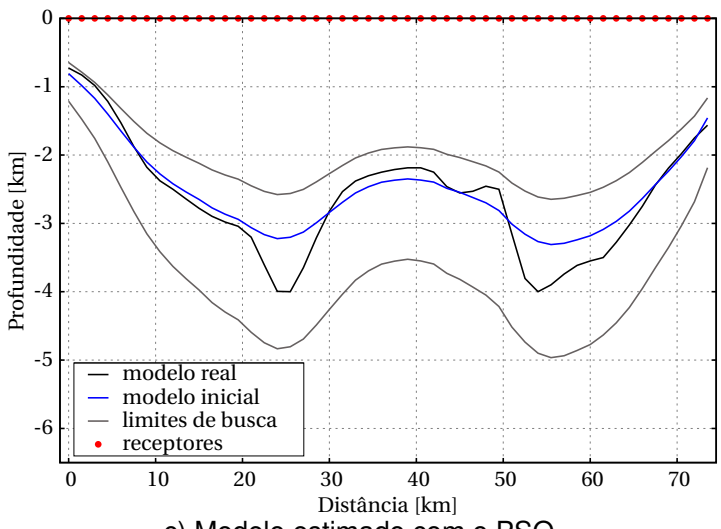

c) Modelo estimado com o PSO.

Figura 8: Resultados para o caso das anomalias contaminadas com ruido branco.

of basement relief using approximate equality constraints on depths.: Geophysics, 62, 1745-1757.

Chakravarthi, V., 1995, Gravity interpretation of nonoutcropping sedimentary basins in which the density contrast decreases parabolically with depth.: Pure Appl. Geophys., 145, 327-335.

Chakravarthi, V., S. Singh, e G. Babu, 2001, INVER2DBASE-a program to compute basement depths of density interfaces above which the density contrast varies with depth.: Comput. Geosci., 27, 1127-1133.

Clerc, M., 1999, The swarm and queen: towards a deterministic and adaptive particle swarm optimization.: Proceedings of the IEEE Congress on Evolutionary Computation., 1951-1957.

Engelbrecht, A., 2007, Computational inteligence an introduction: John Wiley Sons, The Atrium, Southern Gate, Chichester, West Sussex PO19 8SQ, England, 2007.

Holland, J., 1975, Adaptation in natural and artificial systems: an introductory analysis with applications to biology, control, and artificial intelligence.: Michigan: University Michigan Press.

Ingber, L. e B. Rosen, 1992, Genetic algorithms and very fast simulated reannealing: a comparison.: Mathematical and Computer Modelling., 16, 87-100.

Kennedy, J. e R. Eberhart, 1995, Particle swarm optimization.: IEEE International Conference on Neural Networks., 4, 1942-1948.

Marquardt, D., 1963, An algorithm for least-squares estimation of nonlinear parameters.: J. Soc. Ind. Appl. Math., 11, 431-441.

Nocedal, J. e S. Wright, 1999, Numerical optimization.: 1st edition. Springer, New York.

Press, F., 1968, Earth models obtained by monte carlo inversion.: Journal of Geophysical Research, 73, 52235234.

Sajeva, A., M. Aleardi, B. Galuzzi, E. Stucchi, E. Spadavecchia, e A. Mazzotti, 2017, Comparing the performances of four stochastic optimization methods using analytic objective functions, 1D elastic fullwaveform inversion, and residual static computation.: Geophysical Prospecting., 65, 322-346.

Telford, W., L. Geldart, R. Sheriff, e D. Keys, 1976, Applied geophysics.: Cambridge University Press. 